\title{
Taming of macrophage and microglial cell activation by microRNA-124
}

\author{
Ashley T Conrad ${ }^{1}$, Bonnie N Dittel ${ }^{1}$ \\ ${ }^{\prime}$ BloodCenter of Wisconsin, Blood Research Institute, P.O. Box 2178, Milwaukee, WI 53201-2178, USA \\ Cell Research (2011) 21: 213-216. doi:10.1038/cr.2011.9; published online 11 January 2011
}

Microglial cells are from the myeloid lineage and are the only hematopoietic cell resident to the central nervous system (CNS). They populate the CNS during development and are located throughout the CNS. In the adult, microglial cells are maintained in a quiescent state exhibiting a ramified morphology. These resting microglial cells are characterized by a $\mathrm{CD} 45^{\text {lo }}$ phenotype and express very low levels of the antigen presentation molecules MHC class II, B7.2 and CD40 [1]. Although quiescent, microglial cells are dynamic constantly sending out processes to survey their surrounding microenvironment [2]. Upon encounter with activation signals, microglial cells are able to rapidly respond by changing both morphologically and phenotypically. Morphologically, activated microglial cells become amoeboid and resemble macrophages. Phenotypically, activated microglial cells upregulate MHC class II, B7.2 and CD40 (Figure 1). In addition, microglial cells become phagocytic and migratory able to move rapidly towards specific stimuli [2]. Since activated microglial cells have been implicated in a number of neurological disorders, knowing how they are maintained in the quiescent state is of great interest. A recent paper

Correspondence: Bonnie N Dittel

Tel: 1-414-937-3865

E-mail: bonnie.dittel@bcw.edu by Ponomarev et al. in Nature Medicine [3] unravels this mystery implicating the brain-specific microRNA-124 in regulating the activation state of microglial cells and macrophages.

MicroRNAs are small noncoding RNAs of about 22 nucleotides that regulate gene expression by translational repression or mRNA degradation [4]. MicroRNAs are broadly expressed in both plants and animals with the capacity to regulate diverse biological functions such as development, inflammation and oncogenesis [4]. At least seven brain-specific microRNAs have been described, including microRNA-124, which is thought to play a prominent role in brain development by downregulating hundreds of nonneuronal transcripts and by regulating nervous system-specific alternative splicing [5-7]. The study by Ponomarev and colleagues, now demonstrates the expression of high levels of microRNA-124 in the CNS-resident microglial cell population and presents evidence supporting its role in maintaining their quiescent state [3].

Utilizing a mouse model of multiple sclerosis (MS), experimental autoimmune encephalomyelitis (EAE), they found that at the onset of disease the activated microglial cell population had significantly downregulated microRNA-124 expression [3]. In concordance with this finding, highly activated peripheral macrophages expressed very low levels of microRNA-124. Moreover, microRNA-124 expression was found to be low in prenatal and postnatal microglial cells, which exhibit a quasi-activated state compared to adult microglial cells [3]. Thus, microRNA-124 expression in microglial cells is correlated with microglial cell development and activation status. Furthermore, they systematically showed that microRNA-124 acts as a novel modulator of macrophage activation. Forced expression of microRNA-124 in bone marrow-derived macrophages resulted in the downregulation of activation markers and inhibition of TNF- $\alpha$ and inducible nitric oxide synthase expression. Concurrently, there was an upregulation of both the anti-inflammatory cytokine TGF- $\beta 1$ and markers of alternatively activated macrophages, Arg1 and FIZZ1 [3]. MicroRNA-124 was shown to directly inhibit the production of the transcription factor $\mathrm{C} /$ EBP- $\alpha$, resulting in the downregulation of PU.1 [3, 8]. PU.1 is a master regulator of myeloid differentiation and induces the expression of CD45, CD11b, F4/80 and $\mathrm{MHC}$ class II as well as regulating cell proliferation by upregulating the M-CSF receptor $[9,10]$. These data demonstrate the ability of a single microRNA to regulate the expression of multiple genes in myeloid cells. In support of a role for macrophages and microglial cells in the onset and progression of EAE [11], the administration 


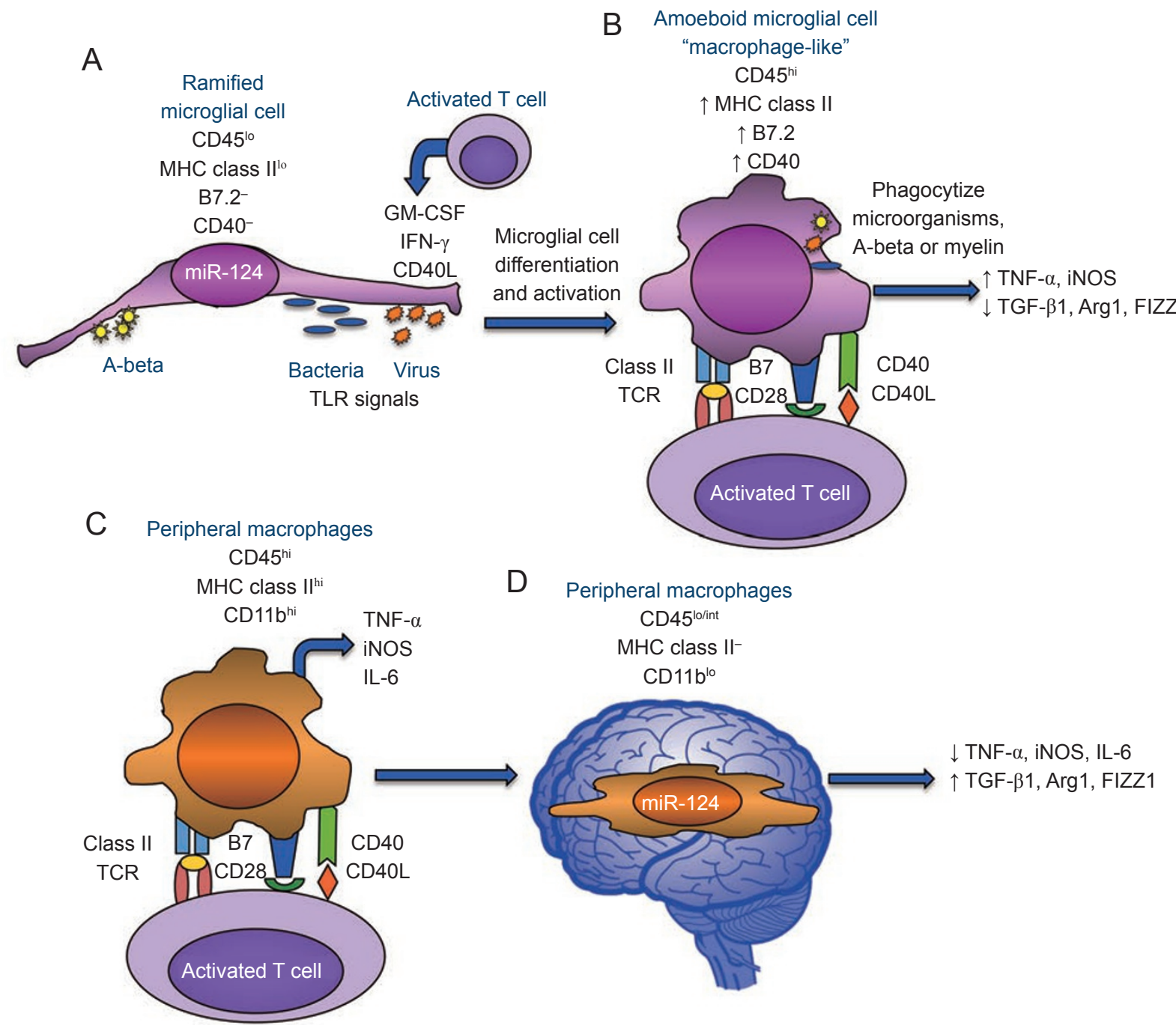

Figure 1 Regulation of the activation state of microglial cells and peripheral macrophages in the CNS via microRNA-124. (A) Within the normal CNS microenvironment, microglial cells express high levels of microRNA-124 causing them to be maintained in a quiescent state characterized by a CD45 ${ }^{\mathrm{lo}}, \mathrm{MHC}$ class $\mathrm{II}^{\mathrm{lo}}, \mathrm{B} 7.2^{-}$and $\mathrm{CD} 40^{-}$phenotype. Microglial cells can be activated by a variety of inflammatory stimuli that includes soluble and cell surface signals that can be delivered by antigenspecific activated T cells (e.g. GM-CSF, IFN- $\gamma$, CD40), microorganisms (TLR signaling by bacteria and viruses) or A-beta. (B) Microglial cell activation occurs rapidly characterized by a morphological transformation from a ramified to an amoeboid cell phenotype. They also acquire an activated phenotype characterized by the upregulation of the cell surface markers CD45, MHC class II, B7.2 and CD40. Activated microglial cells also become phagocytic and efficient antigen presenting cells producing the proinflammatory mediators TNF- $\alpha$ and iNOS. Microglial cell activation is accompanied by downregulation of microRNA-124, which leads to a subsequent increase in the expression of C/EBP- $\alpha$ and PU.1. PU.1, a master regulator of macrophage cell differentiation and proliferation, plays a prominent role in the activation phenotype of microglial cells. (C) Macrophages that migrate into the CNS from the periphery express high levels of C/EBP- $\alpha$ and PU.1, thus, they exhibit an activated phenotype characterized by high levels of expression of CD45, MHC class II, CD11b and produce TNF- $\alpha$, iNOS and IL-6. (D) Once in the CNS, peripheral macrophages respond to the CNS microenvironment and upregulate microRNA-124 leading to reduced expression of CD45, MHC class II and CD11b. This renders the macrophages poor antigen presenting cells. As the macrophages become quiescent, the expression of proinflammatory mediators decreases and they obtain a "M2-like" signature by expressing TGF- $\beta 1$, Arg1 and FIZZ1.

of microRNA-124 was able to prevent EAE when the mice were treated before disease onset and greatly attenuated the severity of disease when administered after onset [3].

The ability to prevent and attenu- 
ate EAE by the administration of microRNA-124 has implications for the treatment of neurodegenerative diseases such as MS and Alzheimer's disease (AD), where microglial cells are thought to play an integral role in the inflammatory process. MS is an autoimmune and inflammatory disease of the CNS characterized by progressive immune-mediated destruction of the myelin sheath and neuronal damage [12]. Although the inflammatory process is believed to be initiated by CD4 T cells, microglial cell activation and macrophage recruitment to the CNS seem to be critical events in the onset, progression and perhaps chronic nature of the disease. The pathogenic $\mathrm{T}$ cells themselves utilize a number of mechanisms to drive the activation of microglial cells (Figure 1). AD is a progressive neurodegenerative disorder with a major pathological feature being the extracellular accumulation of amyloid-beta (A-beta) in the senile plaques. A-beta induces microglial cell activation and chemotactic responses, which is consistent with their presence at sites of A-beta deposition (Figure 1) [13]. The conversion of microglial cells into an activated phenotype results in their production of chemokines, neurotoxic cytokines and reactive oxygen and nitrogen species that are thought to be deleterious to the CNS [13]. Conversely, microglial cells are capable of exerting a neuroprotective role through their release of neurotrophic factors and their ability to clear A-beta by phagocytosis [13]. Rapid microglial cell activation is also driven via innate signals delivered through the toll-like receptor (TLR) family that recognize molecular patterns of microorganisms and viruses (Figure 1). In this situation microglial cell activation would be deemed beneficial due to enhanced phagocytic and antigen presentation capabilities that are needed to drive an immune response required to clear the pathogens (Figure 1). Thus, the ability to modulate microglial cell responses as a therapeutic intervention in neurodegenerative and other diseases of the CNS is of great interest. The recent finding that microRNA-124 can also keep microglial cells in an inactive state and tame macrophage activation [3], makes it an ideal candidate as a novel therapeutic target for the modulation of microglial cell and macrophage activity. Interestingly, the uptake of liposomes containing microRNA- 124 by dendritic cells had no direct effect on their phenotype and they seemed to rapidly degrade the RNA rendering it nonfunctional [3]. As with dendritic cells, microRNA-124 was not detectable in $\mathrm{T}$ and $\mathrm{B}$ cells of microRNA-124 treated mice [3]. These later findings are important because they indicate that therapeutic treatment with microRNA-124 would likely not affect normal immune function.

The discovery that microglial cells of hematopoietic origin express microRNA-124, known to be CNS-specific, suggests that the CNS microenvironment drives the expression of microRNA-124. Interestingly, co-culture of bone marrow-derived macrophages with either a neuronal or astroglial cell line was shown to induce the expression of microRNA-124 in the macrophages driving the cells into a deactivated phenotype [3]. Whether a soluble factor or cell-cell contact is required for the upregulation of microRNA-124 in macrophages and microglial cells is not known. The concept that neurons can tame microglial cell activity has been previously demonstrated [14]. As an example, the absence of CD200 expression on neurons led to the presence of microglial cells that exhibited an activated phenotype and the EAE disease course was accelerated in these same mice [15]. Since CD200R is expressed by microglial cells, it is thought that cell-cell contact with neurons is required for the maintenance of their quiescent phenotype. Interactions between neurons and microglial cells are of primary interest in aging since many neurodegenerative diseases are associated with the aged brain and during aging microglial cells undergo progressive activation [14]. In the aged brain, injured neurons likely interact or communicate with microglial cells, leading to their activation that then in turn contribute to neurodegneration by a variety of mechanisms including release of ATP and soluble factors or the loss of inhibitor molecules controlling their activation state [14]. Thus, it will be interesting to study whether changes in microRNA-124 in the CNS correlate with aging and neurodegeneration. Hence, the discovery that microRNA-124 is a key player in taming the extent of microglial and macrophage activation has the potential to have broad implications in a variety of inflammatory disorders in and out of the CNS.

\section{References}

1 Ponomarev ED, Shriver LP, Maresz K, Dittel BN. Microglial cell activation and proliferation precedes the onset of CNS autoimmunity. $J$ Neurosci Res 2005; 81:374-389.

2 Nimmerjahn A, Kirchhoff F, Helmchen F. Resting microglial cells are highly dynamic surveillants of brain parenchyma in vivo. Science 2005; 308:1314-1318.

3 Ponomarev ED, Veremeyko T, Barteneva N, Krichevsky AM, Weiner HL. MicroRNA-124 promotes microglia quiescence and suppresses EAE by deactivating macrophages via the $\mathrm{C} / \mathrm{EBP}$ alpha-PU.1 pathway. Nat Med 2010 Dec 5; doi:10.1038/nm.2266

4 Bartel DP. MicroRNAs: genomics, biogenesis, mechanism, and function. Cell 2004; 116:281-297.

5 Sempere LF, Freemantle S, Pitha-Rowe I, et al. Expression profiling of mammalian microRNAs uncovers a subset of brain-expressed microRNAs with possible roles in murine and human neuronal differentiation. Genome Biol 2004; 5:R13.

6 Lim LP, Lau NC, Garrett-Engele P, et al. Microarray analysis shows that some microRNAs downregulate large numbers of target mRNAs. Nature 2005; 433:769-773.

7 Makeyev EV, Zhang J, Carrasco MA, Maniatis T. The MicroRNA miR-124 
promotes neuronal differentiation by triggering brain-specific alternative pre-mRNA splicing. Mol Cell 2007; 27:435-448.

8 Zhang P, Iwasaki-Arai J, Iwasaki H, et al. Enhancement of hematopoietic stem cell repopulating capacity and self-renewal in the absence of the transcription factor C/EBP alpha. Immunity 2004; 21:853-863.

9 Celada A, Borras FE, Soler C, et al. The transcription factor PU.1 is involved in macrophage proliferation. J Exp Med
1996; 184:61-69.

10 Nishiyama C, Nishiyama M, Ito T, et al. Functional analysis of PU.1 domains in monocyte-specific gene regulation. FEBS Lett 2004; 561:63-68.

11 Heppner FL, Greter M, Marino D, et al. Experimental autoimmune encephalomyelitis repressed by microglial paralysis. Nat Med 2005; 11:146-152.

12 Sospedra M, Martin R. Immunology of multiple sclerosis. Annu Rev Immunol 2005; 23:683-747.

13 Rogers J, Strohmeyer R, Kovelowski
CJ, Li R. Microglia and inflammatory mechanisms in the clearance of amyloid beta peptide. Glia 2002; 40:260269.

14 Lucin KM, Wyss-Coray T. Immune activation in brain aging and neurodegeneration: too much or too little? Neuron 2009; 64:110-122.

15 Hoek RM, Ruuls SR, Murphy CA, et al. Down-regulation of the macrophage lineage through interaction with OX2 (CD200). Science 2000; 290:17681771. 